\title{
Fast Quadrature Techniques for Retarded Potentials Based on TT/QTT Tensor Approximation
}

\author{
Boris Khoromskij • Stefan Sauter • Alexander Veit
}

\begin{abstract}
We consider the Galerkin approach for the numerical solution of retarded boundary integral formulations of the three dimensional wave equation in unbounded domains. Recently smooth and compactly supported basis functions in time were introduced which allow the use of standard quadrature rules in order to compute the entries of the boundary element matrix. In this paper, we use TT and QTT tensor approximations to increase the efficiency of these quadrature rules. Various numerical experiments show the substantial reduction of the computational cost that is needed to obtain accurate approximations for the arising integrals.
\end{abstract}

2010 Mathematical subject classification: 65F30; 65F50; 65N38; 65F10.

Keywords: multi-dimensional problems; tensor approximation; quantized representation of vectors; model reduction; retarded potentials; 3D wave equation; quadrature rules.

\section{Introduction}

Acoustic and electromagnetic scattering problems in three dimensions have a wide range of practical applications in physics and engineering. An important model problem for the development of efficient and accurate numerical methods for such types of time-dependent physical applications is the three-dimensional wave equation in unbounded exterior domains. Here, boundary element methods show their natural strength, reducing the problem in the unbounded domain to integral equations on the bounded surface of the scatterer.

The efficient numerical solution of such retarded boundary integral equations has gained growing attention in the last years. Existing approaches include methods based on convolution quadrature (cf. [3, 4, 5, 11, 12]) and methods based on bandlimited interpolation and extrapolation (cf. [32, 33, 34, 36]). Here, we consider a Galerkin method in order to discretize the integral equations in space and time (cf. $[2,6,8,9]$ ). It can be shown that the corresponding space-time variational formulation in this approach satisfies a coercivity

The third author gratefully acknowledges the support given by SNF, No. PDFMP2_127437/1

Boris Khoromskij

Max-Planck-Institut für Mathematik in den Naturwissenschaften, Inselstr. 22-26, 04103 Leipzig, Germany

E-mail: bokh@mis.mpg.de.

Stefan Sauter

Institut für Mathematik, Universität Zürich, Winterthurerstr. 190, CH-8057 Zürich, Switzerland

E-mail: stas@math.uzh.ch.

Alexander Veit

Institut für Mathematik, Universität Zürich, Winterthurerstr. 190, CH-8057 Zürich, Switzerland

E-mail: alexander.veit@math.uzh.ch. 
property which ensures the unconditional stability of conforming Galerkin schemes. Furthermore, this approach is very flexible with regard to the use of variable time stepping and spatially curved scatterers. The standard Galerkin approach uses piecewise polynomial basis functions in time. The drawback of the method in this case is that due to the retarded time argument the domain for the spatial integration is the intersection of (possibly curved) pairs of surface panels with the discrete light cone. The stable numerical handling of these intersections is complicated even for flat panels and might be intractable for curved surface patches. We refer to $[7,21,29]$ for examples of quadrature schemes tailored to this problem.

In [26] smooth and compactly supported basis functions in time were introduced. This choice circumvents the problem of integrating on the complicated intersections of the discrete light cone with the spatial surface mesh and allows to apply standard quadrature rules to compute the entries of the boundary element matrix. Due to the compact support of the basis functions the sparsity of the system matrix is maintained. On the other hand this leads to $C^{\infty}$ but, in general, non-analytic integrands, which makes the quadrature problem more difficult. In general, more quadrature points have to be used as for analytic integrands as they arise, e.g., for boundary element methods applied to elliptic boundary value problems.

In this paper we therefore address the problem how to efficiently evaluate the arising integrals using tensor Gauss quadrature and TT/QTT approximation. Note, that other techniques such as sparse, possibly adaptive quadrature also have the potential to be applied to this problem. Preliminary test with sparse grid quadrature indicates, that our TT/QTT approach is preferable for this class of problems, especially if high accuracies are needed. However an asymptotic complexity analysis still has to be done.

The integrals which define the entries of the block system matrix are defined over pairs of surface panels. They are transformed to the reference triangle in Euclidean space and by applying simplex coordinates the quadrature problems boils down to the approximation of an integral over the four-dimensional unit cube. A tensor quadrature rule applied to these integrals leads to a four dimensional tensor A of size $N \times N \times N \times N$, whose entries are the values of the integrand evaluated at the different quadrature points.

To reduce the storage and computational costs to handle this large data array, we apply the methods of tensor approximation based on the idea of separation of variables. There are various tensor-product formats which allow the low parametric representation of highdimensional data. The most commonly used are the canonical, Tucker formats as well as the class of so-called matrix product states (MPS) representations [35, 30, 31] commonly used in high-dimensional quantum computations (see survey paper [16] for more details). Recently these types of tensor formats have attracted much attention in the community of numerical analysis. In particular, the hierarchical Tucker [13], the tensor train (TT) [23] and the tensor chain (TC) [17] formats were considered. In the following we make use of the TT format applied to both the initial fourth order tensor and to its quantized-TT (QTT) representation. Such representations allow to reduce the asymptotical storage and computational costs of certain bilinear tensor operations from $O\left(N^{4}\right)$ to $O\left(r^{2} N\right)$ or even to $O\left(r^{2} \log N\right)$ (avoiding the dependence on the grid-size), where $r$ is the small rank parameter, characterizing the separability properties of the target tensor A. Notice that the hierarchical Tucker format was recently applied in the same spirit to computation of certain multivariate integrals arising in boundary element methods [1].

Various numerical experiments show that these tensors have usually a low rank representation in TT and QTT format which reduces the storage and computational cost substantially. The evaluation of the quadrature then corresponds to a simple scalar product of the 
TT/QTT representation of $\mathbf{A}$ and a rank-1 tensor containing the weights of the quadrature rule. This evaluation can be performed considerably faster compared to the standard approach. In order to compute the TT/QTT approximation of A directly, without computing A itself, we apply a TT cross approximation scheme (cf. [24]) in the QTT format. This further reduces the computational cost, since considerably less evaluations of the integrand are required. We perform numerical experiments to show the efficiency of this scheme in our case.

Note that our sparse approximation method for high-dimensional quadrature problems is by no means restricted to the retarded potential integral equation but, potentially, can be applied to a much larger class of problems. We restricted to this application because quadrature is the major bottleneck for the direct discretization of retarded potentials.

\section{Problem Setting}

Let $\Omega \subset \mathbb{R}^{3}$ be a Lipschitz domain with boundary $\Gamma$. We consider the homogeneous wave equation

$$
\partial_{t}^{2} u-\Delta u=0 \text { in } \Omega \times[0, T]
$$

with initial conditions

$$
u(\cdot, 0)=\partial_{t} u(\cdot, 0)=0 \text { in } \Omega
$$

and Dirichlet boundary conditions

$$
u=g \text { on } \Gamma \times[0, T]
$$

on a time interval $[0, T]$ for $T>0$. In applications, $\Omega$ is often the unbounded exterior of a bounded domain. For such problems, the method of boundary integral equations is an elegant tool where this partial differential equation is transformed to an equation on the bounded surface $\Gamma$. We employ an ansatz as a single layer potential for the solution $u$

$$
u(x, t):=S \phi(x, t):=\int_{\Gamma} \frac{\phi(y, t-\|x-y\|)}{4 \pi\|x-y\|} d \Gamma_{y}, \quad(x, t) \in \Omega \times[0, T]
$$

with unknown density function $\phi . S$ is also referred to as retarded single layer potential due to the retarded time argument $t-\|x-y\|$ which connects time and space variables.

The ansatz (2.2) satisfies the wave equation (2.1a) and the initial conditions (2.1b). Since the single layer potential can be extended continuously to the boundary $\Gamma$, the unknown density function $\phi$ is determined such that the boundary conditions (2.1c) are satisfied. This results in the boundary integral equation for $\phi$,

$$
\int_{\Gamma} \frac{\phi(y, t-\|x-y\|)}{4 \pi\|x-y\|} d \Gamma_{y}=g(x, t) \quad \forall(x, t) \in \Gamma \times[0, T] .
$$

In order to solve this boundary integral equation numerically we introduce the following space-time variational formulation (cf. $[2,8])$ : Find $\phi$ in an appropriate Sobolev space $V$ such that

$$
\int_{0}^{T} \int_{\Gamma} \int_{\Gamma} \frac{\dot{\phi}(y, t-\|x-y\|) \zeta(x, t)}{4 \pi\|x-y\|} d \Gamma_{y} d \Gamma_{x} d t=\int_{0}^{T} \int_{\Gamma} \dot{g}(x, t) \zeta(x, t) d \Gamma_{x} d t
$$

for all $\zeta \in V$, where we denote by $\dot{\phi}$ the derivative with respect to time. 
Let $V_{\text {Galerkin }}$ be a finite dimensional subspace of $V$ being spanned by $L$ basis functions $\left\{b_{i}\right\}_{i=1}^{L}$ in time and $M$ basis functions $\left\{\varphi_{j}\right\}_{j=1}^{M}$ in space. This leads to the fully discrete ansatz

$$
\phi_{\text {Galerkin }}(x, t)=\sum_{i=1}^{L} \sum_{j=1}^{M} \alpha_{i}^{j} \varphi_{j}(x) b_{i}(t), \quad(x, t) \in \Gamma \times[0, T],
$$

where $\alpha_{i}^{j}$ are the unknown coefficients. Plugging this ansatz in (2.4) and rearranging terms shows that this is equivalent to: Find $\alpha_{i}^{j}$ for $i=1, \ldots, L$ and $j=1, \ldots, M$ such that

$$
\sum_{i=1}^{L} \sum_{j=1}^{M} A_{j, l}^{i, k} \alpha_{i}^{j}=g_{l}^{k} \quad \forall 1 \leqslant k \leqslant L \quad \forall 1 \leqslant l \leqslant M,
$$

where

$$
g_{l}^{k}:=\int_{0}^{T} \int_{\Gamma} \dot{g}(x, t) \varphi_{l}(x) b_{k}(t) d \Gamma_{x} d t
$$

and

$$
A_{j, l}^{i, k}:=\int_{\operatorname{supp}\left(\varphi_{l}\right)} \int_{\operatorname{supp}\left(\varphi_{j}\right)} \varphi_{j}(y) \varphi_{l}(x) \psi_{i, k}(\|x-y\|) d \Gamma_{y} d \Gamma_{x} .
$$

The function $\psi_{i, k}$ contains the time integration and is defined, for $s>0$, by

$$
\psi_{i, k}(s):=\int_{0}^{T} \frac{\dot{b}_{i}(t-s) b_{k}(t)}{4 \pi s} d t
$$

Let $\mathcal{G}:=\left\{\tau_{i}: 1 \leqslant i \leqslant \bar{M}\right\}$ denote a finite element mesh on $\Gamma$ consisting of (possibly curved) triangles. More precisely, we assume that for any $\tau \in \mathcal{G}$, there exists a smooth bijection $\chi_{\tau}: \widehat{\tau} \rightarrow \tau$ from the reference element $\widehat{\tau}:=\operatorname{conv}\left\{(0,0)^{\top},(1,0)^{\top},(1,1)^{\top}\right\}$ to the surface triangle $\tau$. Then, in the solution process, the following quadrature problem arises: For $\tau, \tilde{\tau} \in \mathcal{G}$ and $1 \leqslant j, l \leqslant M$, compute

$$
I_{\tau, \tilde{\tau}}^{i, k}\left(\varphi_{j}, \varphi_{l}\right):=\int_{\tau} \int_{\tilde{\tau}} \varphi_{j}(y) \varphi_{l}(x) \psi_{i, k}(\|x-y\|) d \Gamma_{y} d \Gamma_{x},
$$

where $\varphi_{j}$ and $\varphi_{l}$, typically, are lifted polynomials, i.e., $\varphi_{j} \circ \chi_{\tau}$ and $\varphi_{l} \circ \chi_{\tilde{\tau}}$ are polynomials on $\widehat{\tau}$.

The definition of smooth and compactly supported temporal shape functions was addressed in [26] and is as follows. Let

$$
f(t):= \begin{cases}\frac{1}{2} \operatorname{erf}(2 \operatorname{artanh} t)+\frac{1}{2} & |t|<1 \\ 0 & t \leqslant-1 \\ 1 & t \geqslant 1\end{cases}
$$

and note, that $f \in C^{\infty}(\mathbb{R})$. Next, we will introduce some scaling. For a function $g \in$ $C^{0}([-1,1])$ and real numbers $a<b$, we define $g_{a, b} \in C^{0}([a, b])$ by

$$
g_{a, b}(t):=g\left(2 \frac{t-a}{b-a}-1\right) \text {. }
$$

We obtain a bump function on the interval $[a, c]$ with joint $b \in(a, c)$ by

$$
\rho_{a, b, c}(t):= \begin{cases}f_{a, b}(t) & a \leqslant t \leqslant b \\ 1-f_{b, c}(t) & b \leqslant t \leqslant c \\ 0 & \text { otherwise }\end{cases}
$$


Let us now consider the closed interval $[0, T]$ and $l$ (not necessarily equidistant) timesteps

$$
0=t_{0}<t_{1}<\ldots t_{l-2}<t_{l-1}=T \text {. }
$$

We define $\tau_{i}:=\left[t_{i-1}, t_{i}\right]$ for $i=1, \ldots, l-1$. Then $\mathcal{T}:=\left\{\omega_{i}: 1 \leqslant i \leqslant l-1\right\}$ with

$$
\omega_{1}:=\tau_{1}, \quad \omega_{l}:=\tau_{l-1}, \quad \forall 2 \leqslant i \leqslant l-1 \quad \omega_{i}:=\tau_{i-1} \cup \tau_{i}
$$

defines a cover of $[0, T]$. A smooth partition of unity subordinate to $\mathcal{T}$ then is defined by

$$
\varphi_{1}:=1-f_{t_{0}, t_{1}}, \quad \varphi_{l}:=f_{t_{l-2, l-1}}, \quad \forall 2 \leqslant i \leqslant l-1: \varphi_{i}:=\rho_{t_{i-2}, t_{i-1}, t_{i}} .
$$

Smooth and compactly supported basis functions in time can then be obtained by multiplying these partition of unity functions with suitably scaled Legendre polynomials (cf. [26] for details).

Remark 2.1. In the case of lowest order basis functions in time we have $l=L$ and

$$
b_{i}(t)=\varphi_{i}(t) \quad \text { for } \quad i=1, \ldots, L .
$$

With the above definitions it then holds for $\psi_{i, k}$ as defined in (2.8) that:

1. $\operatorname{supp} \psi_{i, k} \subset\left[t_{k-2}-t_{i}, t_{k}-t_{i-2}\right]$.

2. In particular, $\psi_{i, k}=0$ for $k \leqslant i-2$.

3. Let $\mathcal{R}(\tau, \tilde{\tau}):=[\operatorname{dist}(\tau, \tilde{\tau}), \operatorname{maxdist}(\tau, \tilde{\tau})]$, where $\operatorname{maxdist}(\tau, \tilde{\tau}):=\sup _{(x, y) \in \tau \times \tilde{\tau}}\|x-y\|$. Then,

$$
I_{\tau, \tilde{\tau}}^{i, k}\left(\varphi_{j}, \varphi_{l}\right)=0 \quad \text { if } \quad \mathcal{R}(\tau, \tilde{\tau}) \cap\left[t_{k-2}-t_{i}, t_{k}-t_{i-2}\right]=\emptyset .
$$

For higher order basis functions in time similar results can be obtained. Let

$$
\mathcal{I}(\tau, \tilde{\tau}):=\left\{(i, k) \in\{1,2, \ldots, L\}^{2} \mid I_{\tau, \tilde{\tau}}^{i, k}\left(\varphi_{j}, \varphi_{l}\right) \neq 0\right\}
$$

and, vice versa,

$$
\mathcal{I}(i, k):=\left\{(\tau, \tilde{\tau}) \in \mathcal{G} \times \mathcal{G} \mid I_{\tau, \tilde{\tau}}^{i, k}\left(\varphi_{j}, \varphi_{l}\right) \neq 0\right\} .
$$

Note that the index sets $\mathcal{I}(\tau, \tilde{\tau})$ and $\mathcal{I}(i, k)$ are sparse.

Our goal is, in the following, to approximate $I_{\tau, \tilde{\tau}}^{i, k}\left(\varphi_{j}, \varphi_{l}\right)$ efficiently using TT- and QTTapproximations. For simplicity we assume that we have piecewise constant basis functions in space so that $\operatorname{supp} \varphi_{l}=\tau$ and $\operatorname{supp} \varphi_{k}=\tilde{\tau}$ with $\tau, \tilde{\tau} \in \mathcal{G}$. In general these basis functions are lifted piecewise polynomials and typically of low order. Since the use of such low order basis functions in space will not lead to significantly more oscillatory integrands, we do not expect a severe impact of this more general case on the rank decomposition in TT/QTT format.

Because simplex coordinates transform triangles to squares, integrals of the form $(2.9)$ can be written as

$$
\begin{aligned}
& \int_{\tau} \int_{\tilde{\tau}} \psi_{i, k}(\|x-y\|) d \Gamma_{y} d \Gamma_{x}= \\
& \int_{[0,1]}^{4} \underbrace{4|\tau||\tilde{\tau}| \xi_{x} \xi_{y} \psi_{i, k}\left(\left\|\chi_{\tau}\left(\xi_{x}, \xi_{x} \eta_{x}\right)-\chi_{\tilde{\tau}}\left(\xi_{y}, \xi_{y} \eta_{y}\right)\right\|\right)}_{=: f\left(\xi_{x}, \eta_{x}, \xi_{y}, \eta_{y}\right)} d \eta_{y} d \xi_{y} d \eta_{x} d \xi_{x} .
\end{aligned}
$$


We apply properly scaled tensor Gauss-Legendre quadrature rules for the numerical approximation of the arising integrals over the four-dimensional unit cube. Let $n_{1}, n_{2}, n_{3}, n_{4} \in \mathbb{N}_{>0}$ be the number of Gauss quadrature points in the first/second/ third/fourth dimension with nodes

$$
\left(x_{1, i}\right)_{i=1}^{n_{1}},\left(x_{2, j}\right)_{j=1}^{n_{2}},\left(x_{3, k}\right)_{k=1}^{n_{3}},\left(x_{4, l}\right)_{l=1}^{n_{4}} \in[0,1]
$$

and weights

$$
\left(w_{1, i}\right)_{i=1}^{n_{1}},\left(w_{2, j}\right)_{j=1}^{n_{2}},\left(w_{3, k}\right)_{k=1}^{n_{3}},\left(w_{4, l}\right)_{l=1}^{n_{4}} \in \mathbb{R}
$$

Then,

$$
\begin{aligned}
& \int_{[0,1]^{4}} f\left(\xi_{x}, \eta_{x}, \xi_{y}, \eta_{y}\right) d \eta_{y} d \xi_{y} d \eta_{x} d \xi_{x} \approx \\
& \sum_{i=1}^{n_{1}} \sum_{j=1}^{n_{2}} \sum_{k=1}^{n_{3}} \sum_{l=1}^{n_{4}} w_{1, i} w_{2, j} w_{3, k} w_{4, l} f\left(x_{1, i}, x_{2, j}, x_{3, k}, x_{4, l}\right) .
\end{aligned}
$$

For simplicity and in order to test the QTT approximation we set $n_{1}=n_{2}=n_{3}=n_{4}=: N$ and assume that $N$ is a power of 2. The evaluation of an approximation in the form (2.11) requires $O\left(N^{4}\right)$ additions/multiplications and furthermore $O\left(N^{4}\right)$ function evaluations. Since $f$, or more specifically $\psi_{i, k}$, contains itself an integral, such function evaluations might be expensive. Due to the non-analyticity of $f$ and the need to compute the integrals (2.10) accurately in order to obtain stable solutions of the time-domain boundary integral equations, we need a medium number of quadrature points in each direction. Thus, depending on the required accuracy of the approximation, the quadrature problem can become costly. Therefore the question arises if the right hand side in (2.11) can be evaluated more efficiently. For this purpose we will investigate, in the following, the TT and QTT low rank approximations to the fourth order tensor $\mathbf{A}=[A(i, j, k, l)]$ defined entrywise by

$$
A(i, j, k, l)=f\left(x_{1, i}, x_{2, j}, x_{3, k}, x_{4, l}\right), \quad(i, j, k, l) \in\{1, \ldots, N\}^{4} .
$$

Note that for the singular case, where $\operatorname{dist}(\tau, \tilde{\tau})=0$, regularizing coordinate transforms have to be applied to remove the singularity of the kernel function (cf. [28], [25]). In this case, the transformed integral is a sum of integrals over the four-dimensional unit cube and our compression method can be applied also to these cases. For simplicity we restrict in this paper to the approximation of the regular integrals.

\section{Tensor Approximation of $I_{\tau, \tilde{\tau}}^{i, j}\left(\varphi_{j}, \varphi_{l}\right)$}

In the following we apply the matrix-product states (MPS) type tensor representations in the form of tensor train (TT) and quantized-TT (QTT) formats to represent sparsely the fourth order coefficients tensor arising in the quadrature approximation of the above integrals (see (2.11)).

\subsection{Matrix-product states (MPS) tensor formats}

A tensor of order $d$ is defined as an element of finite dimensional tensor-product Hilbert space $\mathbb{W}_{\mathbf{n}} \equiv \mathbb{W}_{\mathbf{n}, d}$ of the $d$-fold, $N_{1} \times \ldots \times N_{d}$ real-valued arrays, and equipped with the Euclidean 
(Frobenius) scalar product $\langle\cdot, \cdot\rangle: \mathbb{W}_{\mathbf{n}} \times \mathbb{W}_{\mathbf{n}} \rightarrow \mathbb{R}$. Each tensor in $\mathbb{W}_{\mathbf{n}}, \mathbf{n}=\left(N_{1}, \ldots, N_{d}\right)$, can be represented componentwise,

$$
\mathbf{A}=\left[A\left(i_{1}, \ldots, i_{d}\right)\right] \quad \text { with } \quad i_{\ell} \in I_{\ell}:=\left\{1, \ldots, N_{\ell}\right\},
$$

where for the ease of presentation, we mainly consider the equal-size tensors, i.e., $N_{\ell}=N$ $(\ell=1, \ldots, d)$. We call the elements of $\mathbb{W}_{\mathbf{n}}=\mathbb{R}^{I_{1} \times \ldots \times I_{d}}$ as $N$ - $d$ tensors. The dimension of the tensor-product Hilbert space $\mathbb{W}_{\mathbf{n}}$ scales exponentially in $d$, $\operatorname{dim} \mathbb{W}_{\mathbf{n}, d}=N^{d}$ implying exponential storage cost for a general $N-d$ tensor.

In our application the quadrature coefficients for approximating $I_{\tau, \tilde{\tau}}^{i, k}\left(\varphi_{j}, \varphi_{l}\right)$ constitute the $N \times N \times N \times N$ tensor $\mathbf{A}$ of order 4 as in (2.12), requiring $N^{4}$ storage size. Hence, in the case of multiple computations of a tensor and high numerical cost of evaluation a single entry, the calculations become nontractable already for $N$ of order several tens.

The MPS representation of a $d$-th order tensor reduces the complexity of storage to $O\left(d r^{2} N\right)$, where $r$ is the maximal mode rank [35, 30]. The MPS tensor approximation was proved to be efficient in high-dimensional electronic/molecular structure calculations, in quantum computing and in stochastic PDEs (see survey paper [16] for more details). In the recent mathematical literature the various versions of MPS tensor decomposition were discovered as the hierarchical Tucker [13], the tensor train (TT) [23] and the tensor chain (TC) [17] formats. In the following we make use of the TT format applied to both the initial $N-d$ tensor and to its quantized representation (quantics-TT).

Definition 3.1. (Tensor chain/train format) For a given rank parameter $\mathbf{r}=\left(r_{0}, \ldots, r_{d}\right)$, and the respective index sets $J_{\ell}=\left\{1, \ldots, r_{\ell}\right\}(\ell=0,1, \ldots, d)$, with the periodicity constraints $J_{0}=J_{d}$ (i.e., $r_{0}=r_{d}$ ), the rank-r TC format contains all elements $\mathbf{A}=\left[A\left(i_{1}, \ldots, i_{d}\right)\right] \in \mathbb{W}_{\mathbf{n}}$ which can be represented as the chain of contracted products of 3 -tensors over the $d$-fold product index set $J:=\times_{\ell=1}^{d} J_{\ell}$

$$
A\left(i_{1}, \ldots, i_{d}\right)=\sum_{\alpha_{1} \in J_{1}} \cdots \sum_{\alpha_{d} \in J_{d}} A^{(1)}\left(\alpha_{d}, i_{1}, \alpha_{1}\right) A^{(2)}\left(\alpha_{1}, i_{2}, \alpha_{2}\right) \cdots A^{(d)}\left(\alpha_{d-1}, i_{d}, \alpha_{d}\right) .
$$

In the matrix form we have the entrywise MPS representation

$$
A\left(i_{1}, i_{2}, \ldots, i_{d}\right)=A_{i_{1}}^{(1)} A_{i_{2}}^{(2)} \ldots A_{i_{d}}^{(d)},
$$

where each $A_{i_{\ell}}^{(\ell)}$ is $r_{\ell-1} \times r_{\ell}$ matrix. In the case $J_{0}=J_{d}=\{1\}$, the TC format coincides with TT representation in [23].

The TC/TT format reduces the storage cost of a $N$-d tensor to $O\left(d r^{2} N\right), r=\max r_{\ell}$. The important multilinear algebraic operations with TT tensors can be implemented with linear complexity scaling in $d$ and $N$. In particular, for the Hadamard product we have

$$
\mathbf{Z}=\mathbf{X} \circ \mathbf{Y}: \quad Z^{(k)}\left(i_{k}\right)=X^{(k)}\left(i_{k}\right) \otimes Y^{(k)}\left(i_{k}\right)
$$

implying the formatted representation of the scalar product (in $O\left(d r^{3} N\right) \ll N^{d}$ operations)

$$
\langle\mathbf{X}, \mathbf{Y}\rangle=\langle\mathbf{X} \circ \mathbf{Y}, \mathbf{1}\rangle \text {. }
$$

\subsection{Quantized-TT (QTT) Approximation of $N-d$ tensors}

Further reduction of the asymptotic storage complexity can be based on the so-called quantizedTT (QTT) representation obtained from the initial $N \times N \times N \times N$ tensor by simple folding 
(reshaping) to a higher dimensional $2 \times \ldots \times 2$ array. It was shown in [17] that the computational gain of the QTT representation is due to the good separability properties of quantized images on a large class of function related tensors. In our application we found numerically the low rank TT/QTT approximations for arising 4th order tensors, indicating nearly the same data compression for both formats. However, the important motivation to use the QTT representation is due to the high efficiency of the QTT-cross approximation scheme ensured by the small mode size (in fact, equals to 2) of the quantized tensors.

We suppose that $N=2^{L}$ with some $L=1,2, \ldots$. The next definition introduces the folding of $N$ - $d$ tensors into the elements (quantized $2 \times \ldots \times 2$ tensors) of an auxiliary $D$-dimensional tensor space with $D=d \log _{2} N$.

Definition 3.2. ([17]) Introduce the binary folding transform of degree $2 \leqslant L$,

$$
\mathcal{F}_{d, L}: \mathbb{W}_{\mathbf{n}, d} \rightarrow \mathbb{W}_{\mathbf{m}, d L}, \quad \mathbf{m}=\left(\mathbf{m}_{1}, \ldots, \mathbf{m}_{d}\right), \quad \mathbf{m}_{\ell}=\left(m_{\ell, 1}, \ldots, m_{\ell, L}\right),
$$

with $m_{\ell, \nu}=2$ for $\nu=1, \ldots, L,(\ell=1, \ldots, d)$, that reshapes the initial $\mathbf{n}-d$ tensor in $\mathbb{W}_{\mathbf{n}, d}$ to elements of the quantized space $\mathbb{W}_{\mathbf{m}, d L}$ as follows:

(A) For $d=1$ a vector $\mathbf{X}=[X(i)]_{i \in I} \in \mathbb{W}_{N, 1}$, is reshaped to the element of $\mathbb{W}_{2, L}$ by

$$
\mathcal{F}_{1, L}: \mathbf{X} \rightarrow \mathbf{Y}=[Y(\mathbf{j})]:=[X(i)], \quad \mathbf{j}=\left\{j_{1}, \ldots, j_{L}\right\},
$$

with $j_{\nu} \in\{1,2\}$ for $\nu=1, \ldots, L$. For fixed $i, j_{\nu}=j_{\nu}(i)$ is defined by $j_{\nu}-1=C_{-1+\nu}$, where the $C_{-1+\nu}$ are found from the binary representation of $i-1$,

$$
i-1=C_{0}+C_{1} 2^{1}+\cdots+C_{L-1} 2^{L-1} \equiv \sum_{\nu=1}^{L}\left(j_{\nu}-1\right) 2^{\nu-1} .
$$

(B) For $d>1$ the construction is similar.

Notice that the folding transform $\mathcal{F}_{d, L}$ is the linear isometry between $\mathbb{W}_{N, d}$ and $\mathbb{W}_{2, d L}$ (see [17]).

Remark 3.1. Every 2- $d L$ tensor in the quantics space $\mathbb{W}_{2, d L}$ can be represented (approximated) in the low rank TT format. This leads to the so-called QTT representation of $N-d$ tensors. Assuming that $r_{k} \leqslant r, k=1, \ldots, d L$, the complexity of the QTT representation can be estimated by $O\left(d r^{2} \log N\right)$, providing log-volume asymptotics compared with the volume size of the initial tensor $O\left(N^{d}\right)$.

\subsection{Sketch of numerical TT/QTT approximation}

The manifold [14] of rank-r TT tensors in $\mathbb{W}_{\mathbf{n}}$ is known to be closed in the Frobenius norm $[24]$.

From the computational point of view, one of the most attractive features of TT format is the following: the numerical computation of $r_{k-1} \times r_{k}$ matrices $A_{i_{k}}^{(k)}$ in the TT representation (approximation) of a full format tensor $\mathbf{A}=\left[A\left(i_{1}, \ldots, i_{d}\right)\right]$,

$$
A\left(i_{1}, i_{2}, \ldots, i_{d}\right)=A_{i_{1}}^{(1)} A_{i_{2}}^{(2)} \ldots A_{i_{d}}^{(d)}
$$

can be implemented by a stable SVD-based algorithm (MATLAB Toolbox http://spring. inm.rus.ru/osel). For the completeness of presentation, we sketch the full-to-TT compression algorithm [23], which will be applied in Section 4 to our particular fourth order coefficients tensor. 
Input: a tensor $\mathbf{A}$ of size $n_{1} \times n_{2} \cdots \times n_{d}$ and accuracy bound $\varepsilon>0$.

1: First unfolding: $N_{r}=\prod_{k=2}^{d} n_{k}, M:=\operatorname{reshape}\left(\mathbf{A},\left[n_{1}, N_{r}\right]\right)$.

2: Compute the truncated SVD of $M \approx U \Lambda V$, so that the approximate rank $r$ ensures

$$
\sum_{k=r+1}^{\min \left(n_{1}, N_{r}\right)} \sigma_{k}^{2} \leqslant \frac{\left(\varepsilon \cdot\|\mathbf{A}\|_{F}\right)^{2}}{d-1}
$$

3: Set $A^{(1)}=U, M:=\Lambda V^{T}, r_{1}=r$, and process modes $k=2, \ldots, d-1$.

4: for $k=2$ to $d-1$ do

4a: Construct the next unfolding: $N_{r}:=\frac{N_{r}}{n_{k}}, M:=\operatorname{reshape}\left(M,\left[r n_{k}, N_{r}\right]\right)$.

4b: Compute the truncated SVD of $M \approx U \Lambda V$, so that the approximate rank $r$ ensures

$$
\sum_{k=r+1}^{\min \left(n_{k}, N_{r}\right)} \sigma_{k}^{2} \leqslant \frac{\left(\varepsilon \cdot\|\mathbf{A}\|_{F}\right)^{2}}{d-1}
$$

4c: Set $r_{k}=r$ and reshape the matrix $U$ into a tensor:

$$
A^{(k)}:=\operatorname{reshape}\left(U,\left[r_{k-1}, n_{k}, r_{k}\right]\right)
$$

4d: Recompute $M:=\Lambda V$.

\section{end for}

5: Set $A^{(d)}=M$.

Output: TT cores $A_{k}, k=1, \ldots d$, defining a TT $\varepsilon$-approximation to $\mathbf{A}$.

The above algorithm has the numerical complexity $O\left(n^{d+1}\right)$. In the present paper we directly apply this algorithm to the fourth-order tensor of interest to demonstrate the efficient rank decomposition in the TT format that reduces drastically the storage and computational cost. Moreover, assuming the existence of low-rank TT representation the rank-r TT approximation can be computed by the heuristic algorithm called TT-cross approximation [24] avoiding the "curse of dimensionality" (see the numerical example below). This algorithm also applies to QTT format (QTT-cross approximation).

Remark 3.2. Notice that the QTT approximation of the target $N \times N \times N \times N$ tensor A can be performed by the same decomposition algorithm but applied in the particular setting $n_{k}=2, d=4 \log N$. The rank- $r$ QTT-cross approximation takes the advantage of low cost $O\left(r^{4} \log N\right)$ since, due to the main property of TT-cross algorithm, it calls only for $O\left(r^{2} \log N\right)$ entries of the initial tensor $\mathbf{A}$. In this way, the generation of the full tensor can be avoided by using the rank- $r$ QTT-cross approximation method that requires to compute only few entries (chosen adaptively) of the target tensor. The numerical results show that the compression is comparable with the complete QTT approximation method (see Section 4.6).

\subsection{Computation of $I_{\tau, \tilde{\tau}}^{i, j}\left(\varphi_{j}, \varphi_{l}\right)$ using TT/QTT approximation}

Let us denote the TT and QTT representations of $\mathbf{A}$, defined in (2.12), by $\mathbf{A}_{T T}$ and $\mathbf{A}_{Q T T}$. An approximation of the integral in (2.11) using these representations instead of $\mathbf{A}$ can be obtained by a simple tensor operation in the quantics space $\mathbb{W}_{2, d L}, d=4, L=\log N$, specifically as the scalar product of the rank-1 coefficients tensor $\mathbf{W}=w_{1} \otimes w_{2} \otimes w_{3} \otimes w_{4}$ 
with $\mathbf{A}_{T T}$ or $\mathbf{A}_{Q T T}$. Let

$$
\begin{aligned}
& Q_{G}:=\langle\mathbf{W}, \mathbf{A}\rangle=\sum_{i=1}^{N} \sum_{j=1}^{N} \sum_{k=1}^{N} \sum_{l=1}^{N} w_{1, i} w_{2, j} w_{3, k} w_{4, l} f\left(x_{1, i}, x_{2, j}, x_{3, k}, x_{4, l}\right), \\
& Q_{T T}:=\left\langle\mathbf{W}, \mathbf{A}_{T T}\right\rangle \\
& Q_{Q T T}:=\left\langle\mathbf{W}, \mathbf{A}_{Q T T}\right\rangle
\end{aligned}
$$

denote the quadrature formulas based on the different representations of $\mathbf{A}$. As pointed out in Section 3.1 the cost to evaluate the scalar products $Q_{T T}$ or $Q_{Q T T}$ scales with $O\left(4 r^{3} N\right)$, where $r$ is much smaller than $N$, compared to $O\left(N^{4}\right)$ for the exact evaluation of $Q_{G}$. Therefore the approximations $Q_{T T}$ and $Q_{Q T T}$ can be computed considerably faster, provided that $\mathbf{A}$ has TT and QTT representations with low rank.

Since $\mathbf{A}_{T T}$ and $\mathbf{A}_{Q T T}$ are only approximations of $\mathbf{A}$, the formulas $Q_{T T}$ and $Q_{Q T T}$ introduce additional quadrature errors. An important question therefore is how accurate the approximations $\mathbf{A}_{T T / Q T T}$ have to be, such that the relative errors

$$
E_{G, T T}:=\frac{\left|Q_{G}-Q_{T T}\right|}{\left|Q_{G}\right|} \quad \text { and } \quad E_{G, Q T T}:=\frac{\left|Q_{G}-Q_{Q T T}\right|}{\left|Q_{G}\right|}
$$

are small and the additional error does not affect the accuracy of the quadrature $Q_{G}$.

\section{Numerical Experiments}

In the following, we investigate the compression properties of $\mathbf{A}$ and the accuracy of $Q_{T T}$ and $Q_{Q T T}$ using different triangles and time meshes in order to cover various cases, that might occur during the solution of the discrete system (2.6). Therefore, let

$$
\begin{aligned}
& \tau:=\operatorname{conv}\left\{(0,0,0)^{\mathrm{T}},(1,0,0)^{\mathrm{T}},(1,1,0)^{\mathrm{T}}\right\}, \\
& \tilde{\tau}:=c_{\text {shift }}+\operatorname{conv}\left\{(1,0,0)^{\mathrm{T}},\left(1, \frac{1}{2}, 1\right)^{\mathrm{T}},\left(0,1, \frac{1}{2}\right)^{\mathrm{T}}\right\},
\end{aligned}
$$

with $c_{\text {shift }} \in \mathbb{R}$. These triangles will be used for all numerical experiments. Only $c_{\text {shift }} \in \mathbb{R}$ is variable and will be set individually for each case. Furthermore we will define different time grids for each case consisting of six points $t_{1} \leqslant \ldots \leqslant t_{6} \in \mathbb{R}_{\geqslant 0}$. We then choose basis functions $b(t)$ and $\tilde{b}(t)$ in time such that $\operatorname{supp} b=\left[t_{1}, t_{3}\right]$ and $\operatorname{supp} \tilde{b}=\left[t_{4}, t_{6}\right]$. More precisely, $b$ and $\tilde{b}$ will be the smooth bump functions as defined in Section 2 multiplied with properly scaled Legendre polynomials of degree 1 (cf. [26]), i.e.,

$$
b(t)=\rho_{t_{1}, t_{2}, t_{3}}(t)\left(2 \frac{t-t_{1}}{t_{3}-t_{1}}-1\right) \quad \text { and } \quad \tilde{b}(t)=\rho_{t_{4}, t_{5}, t_{6}}(t)\left(2 \frac{t-t_{4}}{t_{6}-t_{4}}-1\right) .
$$

Thus, the integrals we want to approximate are of the form

$$
I_{\tau, \tilde{\tau}}:=\int_{\tau} \int_{\tilde{\tau}} \psi(\|x-y\|) d \Gamma_{y} d \Gamma_{x}
$$

with

$$
\psi(s):=\int_{0}^{T} \frac{\dot{b}(t-s) \tilde{b}(t)}{4 \pi s} d t
$$




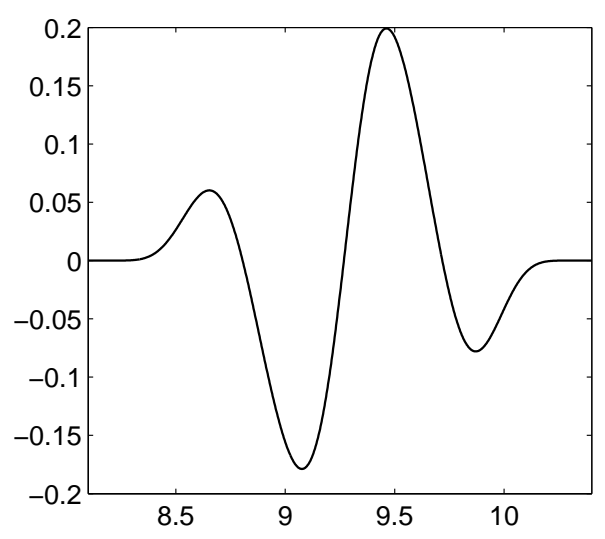

Figure 4.1. $\psi(s)$ for the time grid given in (4.4)

where $s \in \mathbb{R}_{>0}$. Note that

$$
\operatorname{supp} \psi=\left[t_{4}-t_{3}, t_{6}-t_{1}\right] .
$$

We denote the domain of the spatial integration by

$$
S=\left\{z \in \mathbb{R}^{3} \text { s.t. } z=x-y, x \in \tau, y \in \tilde{\tau}\right\}
$$

and define

$$
S_{\min }:=\min _{z \in S}\|z\|=\operatorname{dist}(\tau, \tilde{\tau}), \quad S_{\max }:=\max _{z \in S}\|z\|=\operatorname{maxdist}(\tau, \tilde{\tau}) .
$$

It can be easily seen that the position of triangle $\tilde{\tau}$, i.e. $c_{\text {shift }}$, has to be chosen such that $\left[S_{\min } S_{\max }\right] \cap\left[t_{4}-t_{3}, t_{6}-t_{1}\right] \neq \emptyset$ in order to obtain $I_{\tau, \tilde{\tau}} \neq 0$ (cf. Remark 2.1). In the following we will perform numerical experiments for the following cases:

1. $S_{\min }<t_{4}-t_{3}$ and $S_{\max }<t_{6}-t_{1}$. Here, the domain $S$ is only partially enlighted from one side (cf. Fig. 4.2). The case $S_{\min }>t_{4}-t_{3}$ and $S_{\max }>t_{6}-t_{1}$ leads to similar numerical results in our example and will not be treated separately.

2. $S_{\min }>t_{4}-t_{3}$ and $S_{\max }<t_{6}-t_{1}$. In this case the domain $S$ is completely enlighted (cf. Fig. 4.4).

3. $S_{\min }<t_{4}-t_{3}$ and $S_{\max }>t_{6}-t_{1}$. Here, the discrete light cone is a narrow strip (cf. Fig. 4.6).

4. $S_{\min }$ small. In this case we examine how small distances between the triangles influence the compression rates.

5. At last we consider the case of higher order basis functions in time and therefore a more oscillatory function $\psi$.

Remark 4.1. The following numerical experiments were performed using MATLAB on an Intel Q8200 processor, 4Gb RAM. The TT/QTT approximations of the tensor A were computed using the TT-toolbox 1.0 for MATLAB written by I. Oseledets (http://spring. inm.rus.ru/osel). 


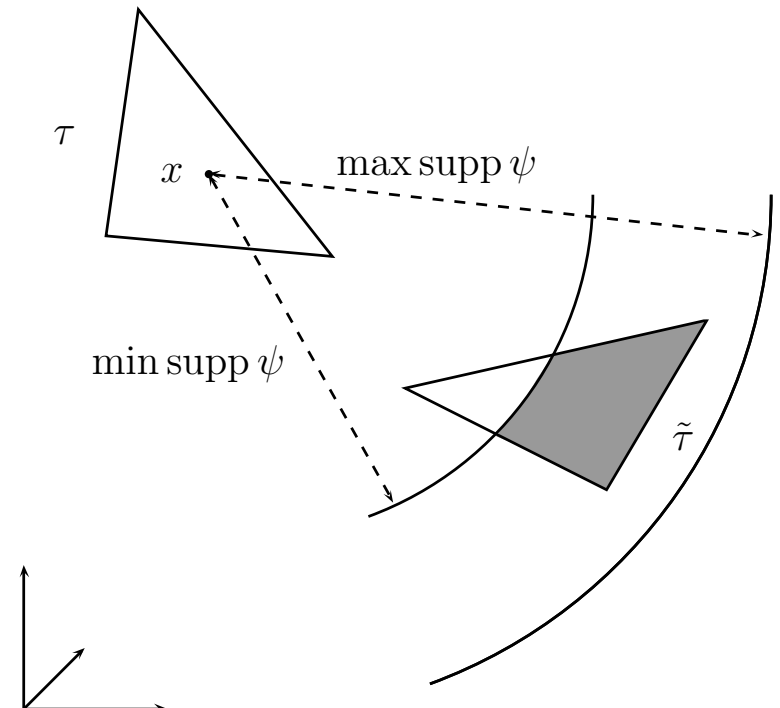

Figure 4.2. Enlighted region for fixed $x \in \tau$ in Case 1

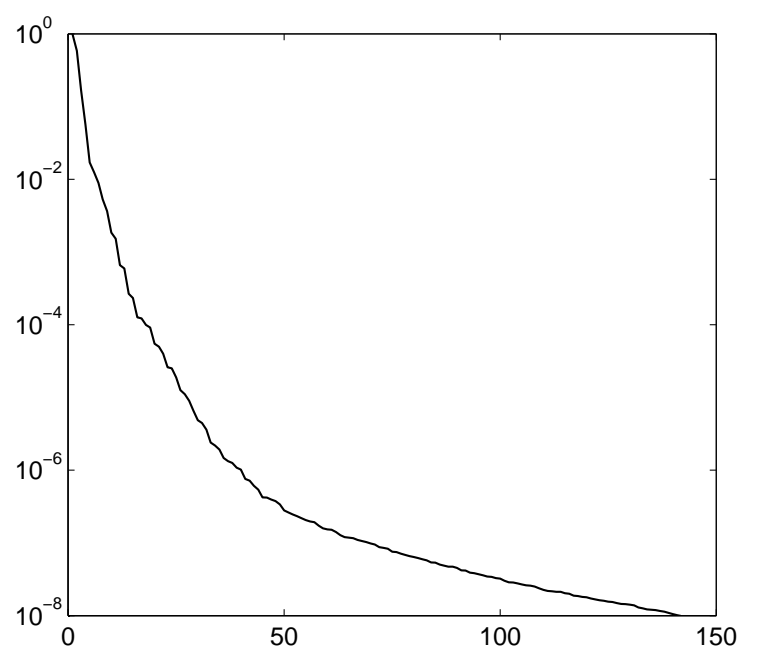

\subsection{Case 1: Partially enlighted integration domain}

For this case we define the time grid

$$
t_{1}=0.6, \quad t_{2}=1.2, \quad t_{3}=1.7, \quad t_{4}=9.8, \quad t_{5}=10.5, \quad t_{6}=11.0
$$

and $c_{\text {shift }}=4.4$ such that $S_{\min } \approx 7.2$ and $S_{\max } \approx 9.6$. This choice of the parameters leads to a situation as illustrated in Fig. 4.2. The integration domain is only partially enlighted from one side, which leads (depending of the choice of $c_{\text {shift }}$ ) to many zero entries in the resulting tensor $\mathbf{A}$. In this example $c_{\text {shift }}$ was chosen such that approximately $50 \%$ of the entries of $\mathbf{A}$ are nonzero.

Since we are mostly concerned with far field integrals we expect that a fixed number of quadrature points leads to a sufficiently high accuracy of the approximations. For the near field integrals we expect that the number of quadrature points has to be asymptotically increased, e.g., in a logarithmic way. However a rigorous theoretical analysis of the influence of the quadrature error on the total discretization error is still open. For the approximation of $I_{\tau, \tilde{\tau}}$ we set $N=32$, i.e., we use 32 Gauss quadrature points in each direction leading to a tensor $\mathbf{A}$ with $\operatorname{size}(\mathbf{A})=32 \times 32 \times 32 \times 32$. In order to compute singular values we reshape A to a matrix $\mathbf{B}$ of size $32^{2} \times 32^{2}$. Note, that in many cases the number of quadrature points can be chosen lower in order to obtain accurate approximations.

The table below shows the efficiency of the TT-approximation $\mathbf{A}_{T T}$ and the QTTapproximation $\mathbf{A}_{Q T T}$ of $\mathbf{A}$. We listed the mean ranks of the corresponding cores for different approximation accuracies. We additionally computed the singular value decomposition of $\mathbf{B}$ and listed the number of relative singular values that are greater than the prescribed accuracy. The decay of the singular values is shown in Fig. 4.3. It can be observed that the ranks of the TT- and QTT-approximation are small, especially for low and medium accuracies. The low ranks in this case could be found also for other configurations of the numerical experiment. In general it can be noticed that the compression in this case is better if many entries of $\mathbf{A}$ are zero or in other words that the enlighted part of the integration domain is small. (That a sparse A however does not necessarily lead to good compression rates can be seen in Section 4.3). 


\begin{tabular}{|c|c|c|c|}
\hline Accuracy & Mean rank of $\mathbf{A}_{T T}$ & Mean rank of $\mathbf{A}_{Q T T}$ & Rel. SV of B \\
\hline $10^{-2}$ & 5.7 & 8.0 & 7 \\
$10^{-3}$ & 9.4 & 15.2 & 12 \\
$10^{-4}$ & 13.0 & 23.1 & 18 \\
$10^{-5}$ & 18.7 & 33.4 & 28 \\
$10^{-6}$ & 25.4 & 45.5 & 41 \\
\hline
\end{tabular}

Table 4.1.

In the next table we compare the time that is needed to compute the approximations $Q_{G}, Q_{T T}$ and $Q_{Q T T}$ for different accuracies of the TT- and QTT-approximation. We assume that $\mathbf{A}, \mathbf{A}_{T T}$, and $\mathbf{A}_{Q T T}$ are given in each case, so that only the different scalar products (3.2)(3.4) have to be evaluated. Furthermore we compute the relative errors $E_{G, T T}$ and $E_{G, Q T T}$ (cf. (3.5)) in order to see the effect of the additional approximation on the quadrature result.

\begin{tabular}{|c|c|c|c|c|c|}
\hline Accuracy & Time $Q_{G}$ & Time $Q_{T T}$ & $E_{G, T T}$ & Time $Q_{Q T T}$ & $E_{G, Q T T}$ \\
\hline $10^{-2}$ & 100 & 1.3 & $2 \cdot 10^{-3}$ & 9.8 & $2 \cdot 10^{-4}$ \\
$10^{-3}$ & 100 & 1.3 & $4 \cdot 10^{-5}$ & 10.1 & $1 \cdot 10^{-4}$ \\
$10^{-4}$ & 100 & 1.4 & $2 \cdot 10^{-6}$ & 10.3 & $6 \cdot 10^{-6}$ \\
$10^{-5}$ & 100 & 1.5 & $1 \cdot 10^{-7}$ & 10.8 & $2 \cdot 10^{-7}$ \\
$10^{-6}$ & 100 & 1.6 & $7 \cdot 10^{-8}$ & 11.2 & $4 \cdot 10^{-8}$ \\
\hline
\end{tabular}

Table 4.2.

It can be seen above that the evaluation of $Q_{T T}$ and $Q_{Q T T}$ is considerably faster than the evaluation of $Q_{G}$ due to the low ranks of $\mathbf{A}_{T T}$ and $\mathbf{A}_{Q T T}$ and the induced low number of arithmetic operations that are needed to compute the corresponding scalar products. Furthermore it can be observed that the errors $E_{G, T T}$ and $E_{G, Q T T}$ are small even for low and medium accuracies of the TT- and QTT-approximation. In this case it is sufficient to determine $\mathbf{A}_{T T}$ and $\mathbf{A}_{Q T T}$ with relatively low accuracy in order to obtain accurate approximations for $Q_{G}$. On the one hand this is advantageous since we benefit from low ranks in this case and on the other hand the computation of $\mathbf{A}_{T T}$ and $\mathbf{A}_{Q T T}$ directly via TT/QTT cross approximation becomes cheaper as well (cf. Section 4.6).

\subsection{Case 2: Completely enlighted integration domain}

For this case we again use the time grid (4.4) and set $c_{\text {shift }}=5.1$ such that $S_{\min } \approx 8.42$ and $S_{\max } \approx 10.28$. We are therefore in the situation where the integration domain $\tau \times \tilde{\tau}$ is completely enlighted (cf. Fig. 4.4). Thus, $\mathbf{A}$ is in general densely populated with no vanishing entries. We set again $N=32$ and compute the mean ranks of the TT- and QTT approximation of $\mathbf{A}$. The decay of the relative singular values of the reshaped matrix $\mathbf{B}$ is shown in Fig. 4.5.

The results of the numerical experiments indicate that the compression rates in this case are very similar to Case 1 . Thus a fully populated tensor $\mathbf{A}$ does not have a severe negative impact on the ranks of $\mathbf{A}_{T T}$ and $\mathbf{A}_{Q T T}$ compared to a situation where the integration domain ist only partially enlighted and similar basis functions in time are used. The next table shows the time that is needed to compute the different approximations of $I_{\tau, \tilde{\tau}}$. Thereby we again 


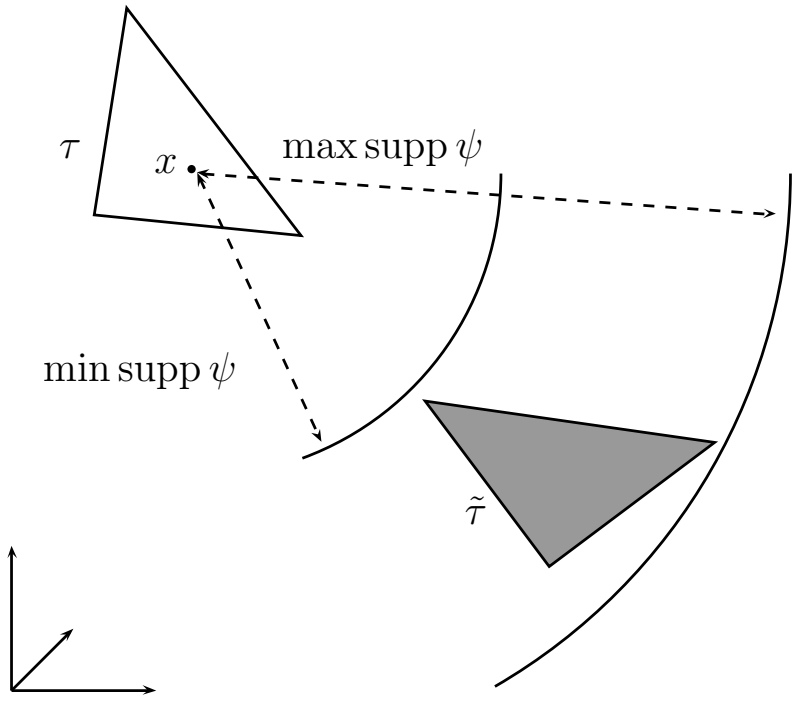

Figure 4.4. Enlighted region for fixed $x \in \tau$ in Case 2

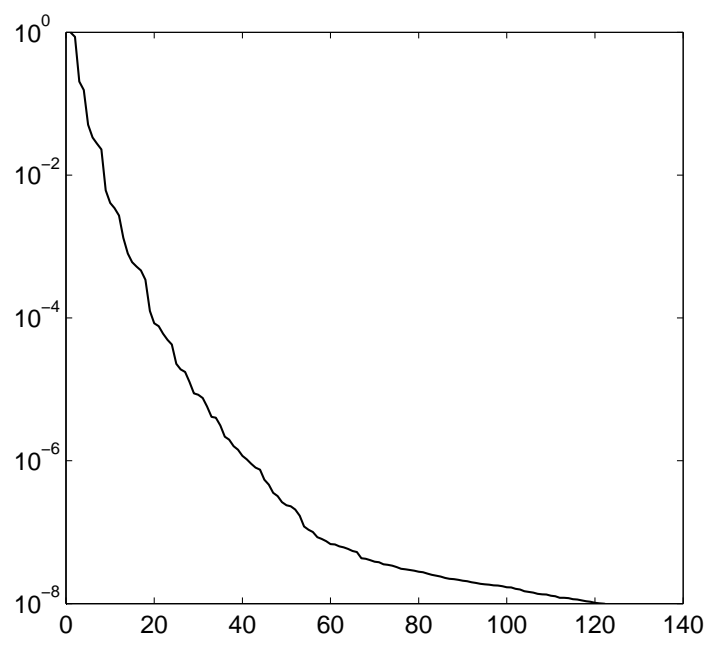

Figure 4.5. Relative singular values of B. Non-zero entries of B: $100 \%$

\begin{tabular}{|c|c|c|c|}
\hline Accuracy & Mean rank of $\mathbf{A}_{T T}$ & Mean rank of $\mathbf{A}_{Q T T}$ & Rel. SV of B \\
\hline $10^{-2}$ & 6.7 & 10.4 & 9 \\
$10^{-3}$ & 9.8 & 18.2 & 14 \\
$10^{-4}$ & 13.4 & 29.1 & 20 \\
$10^{-5}$ & 18.4 & 40.5 & 29 \\
$10^{-6}$ & 25.0 & 53.3 & 42 \\
\hline
\end{tabular}

Table 4.3.

\begin{tabular}{|c|c|c|c|c|c|}
\hline Accuracy & Time $Q_{G}$ & Time $Q_{T T}$ & $E_{G, T T}$ & Time $Q_{Q T T}$ & $E_{G, Q T T}$ \\
\hline $10^{-2}$ & 100 & 1.3 & $7 \cdot 10^{-3}$ & 10.0 & $5 \cdot 10^{-2}$ \\
$10^{-3}$ & 100 & 1.4 & $1 \cdot 10^{-3}$ & 10.3 & $4 \cdot 10^{-4}$ \\
$10^{-4}$ & 100 & 1.4 & $8 \cdot 10^{-5}$ & 10.6 & $4 \cdot 10^{-5}$ \\
$10^{-5}$ & 100 & 1.5 & $3 \cdot 10^{-6}$ & 10.8 & $3 \cdot 10^{-6}$ \\
$10^{-6}$ & 100 & 1.7 & $4 \cdot 10^{-8}$ & 11.3 & $1 \cdot 10^{-8}$ \\
\hline
\end{tabular}

Table 4.4.

assume that $\mathbf{A}, \mathbf{A}_{T T}$ and $\mathbf{A}_{Q T T}$ are given for each accuracy. As expected the evaluation of the scalar product using the TT- and QTT approximation is considerably faster. Furthermore, the relative errors $E_{G, T T}$ and $E_{G, Q T T}$ are, as in the previous case, small for medium accuracies of $\mathbf{A}_{T T}$ and $\mathbf{A}_{Q T T}$.

\subsection{Case 3: Narrow discrete light cone}

Here we want to examine how a narrow discrete light cone, i.e., the support of $\psi$ is a small interval, influences the compression rates. Therefore we consider the time mesh

$$
t_{1}=0.6, \quad t_{2}=0.8, \quad t_{3}=1.0, \quad t_{4}=10.3, \quad t_{5}=10.45, \quad t_{6}=10.7
$$




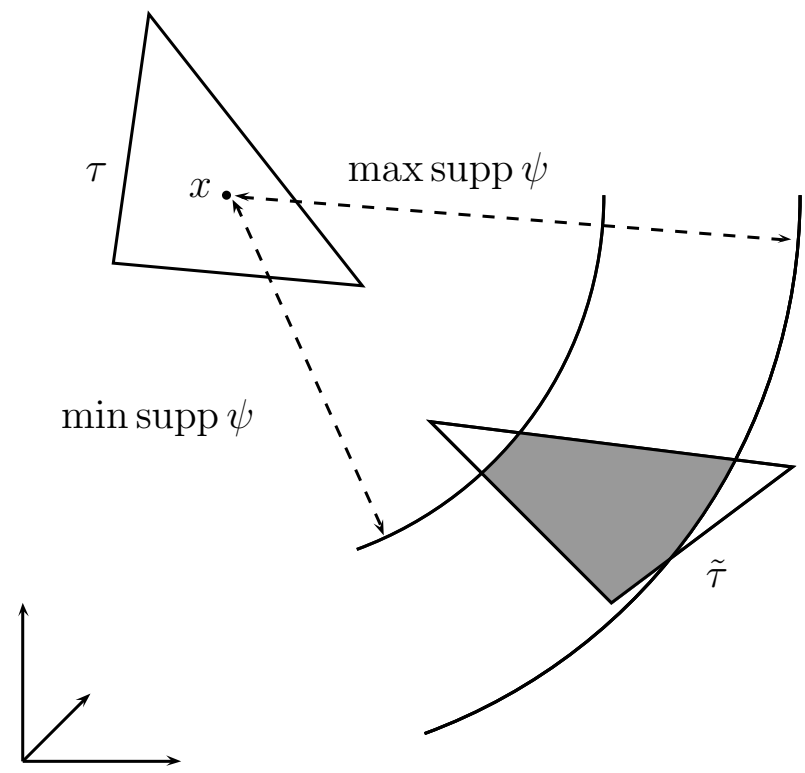

Figure 4.6. Enlighted region for fixed $x \in \tau$ in Case 3

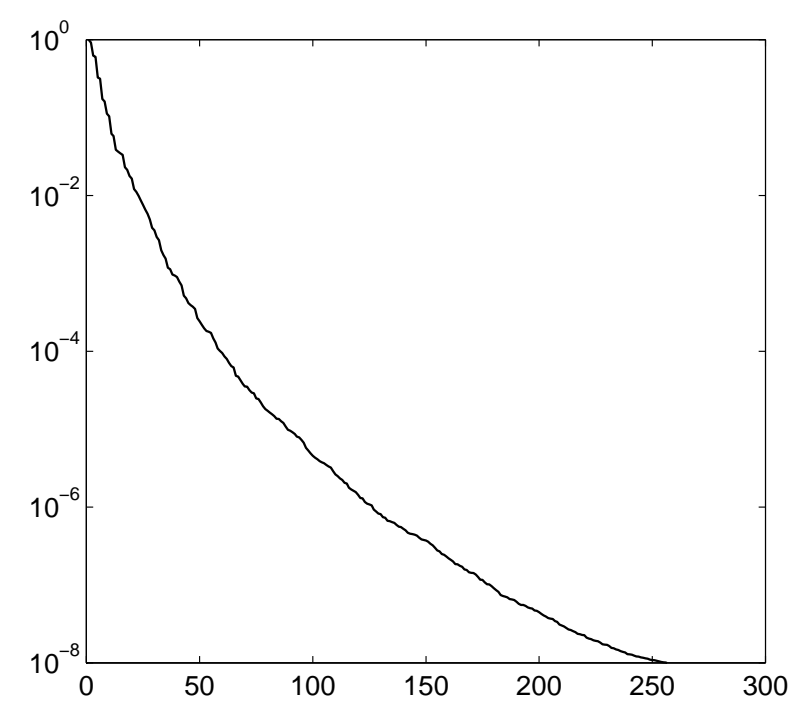

Figure 4.7. Relative singular values of B. Non-zero entries of B: $\approx 64 \%$

such that $\operatorname{supp} \psi=[9.3,10.1]$. Choosing $c_{\text {shift }}=5.4$ leads to the case where $S_{\min }<9.3$ and $S_{\max }>10.1$. We are thus in the situation illustrated in Fig. 4.6. We set again $N=32$ and compute the mean ranks of the TT- and QTT approximation of A which has approximately $64 \%$ nonzero entries. The decay of the relative singular values of the reshaped matrix $\mathbf{B}$ is shown in Fig. 4.7.

\begin{tabular}{|c|c|c|c|}
\hline Accuracy & Mean rank of $\mathbf{A}_{T T}$ & Mean rank of $\mathbf{A}_{Q T T}$ & Rel. SV of B \\
\hline $10^{-2}$ & 14.4 & 21.8 & 23 \\
$10^{-3}$ & 23.3 & 46.8 & 37 \\
$10^{-4}$ & 33.2 & 69.7 & 60 \\
$10^{-5}$ & 44.3 & 97.1 & 89 \\
$10^{-6}$ & 57.0 & 130.1 & 126 \\
\hline
\end{tabular}

Table 4.5.

As one can see in the table above, the compression rates are worse than in the previous cases. This is not surprising since $\psi$ has the same oscillatory behavior as before but varies on a smaller interval. The approximation of the tensor $\mathbf{A}$, which is based on the evaluation of $\psi$ at different points in $\tau \times \tilde{\tau}$ and not only in a narrow strip containing the discrete light cone, is therefore clearly more difficult. This is confirmed by various numerical experiments. The narrower the discrete light cone is, the higher are the mean ranks of the TT- and QTT approximation of $\mathbf{A}$ in general. This case is therefore an example where a more sparse $\mathbf{A}$ does not lead to better compression rates.

Although the mean ranks of $\mathbf{A}_{T T}$ and $\mathbf{A}_{Q T T}$ are larger here than in the previous cases, the compression is still good enough to reduce the computing times of the quadratures considerably. Another effect that can be observed here is, that the errors $E_{G, T T}$ and $E_{G, Q T T}$ decay slower than before. The approximations of $\mathbf{A}$ have therefore to be computed with higher accuracy in order to obtain good approximations of $Q_{G}$. 


\begin{tabular}{|c|c|c|c|c|c|}
\hline Accuracy & Time $Q_{G}$ & Time $Q_{T T}$ & $E_{G, T T}$ & Time $Q_{Q T T}$ & $E_{G, Q T T}$ \\
\hline $10^{-2}$ & 100 & 1.3 & $4 \cdot 10^{-1}$ & 9.0 & $6 \cdot 10^{-1}$ \\
$10^{-3}$ & 100 & 1.4 & $1 \cdot 10^{-2}$ & 9.5 & $2 \cdot 10^{-2}$ \\
$10^{-4}$ & 100 & 1.6 & $1 \cdot 10^{-4}$ & 10.2 & $1 \cdot 10^{-3}$ \\
$10^{-5}$ & 100 & 1.8 & $5 \cdot 10^{-5}$ & 11.1 & $5 \cdot 10^{-5}$ \\
$10^{-6}$ & 100 & 2.1 & $1 \cdot 10^{-6}$ & 12.4 & $1 \cdot 10^{-6}$ \\
\hline
\end{tabular}

Table 4.6.

\subsection{Case 4: Near field integrals}

We now want to test the compression rates in the case where the triangles in (4.2) are close to each other. Since the integrand in (4.2) is weakly singular for $x=y$, the convergence rates of standard quadrature rules deteriorate for $\operatorname{dist}(\tau, \tilde{\tau}) \rightarrow 0$. We examine if low distances between the triangles also have a negative influence on the compression rates of the TT- and QTT-approximation. In order to test this numerically we use the triangles $\tau, \tilde{\tau}$ as before and set $c_{\text {shift }}=1$. In this case we have

$$
\operatorname{dist}(\tau, \tilde{\tau}) \approx 1.44 \quad \text { and } \quad \operatorname{maxdist}(\tau, \tilde{\tau}) \approx 3.20
$$

As time grid we choose

$$
t_{1}=0.6, \quad t_{2}=1.2, \quad t_{3}=1.9, \quad t_{4}=4.2, \quad t_{5}=4.7, \quad t_{6}=5.7,
$$

such that $\operatorname{supp} \psi=[2.3,5.1]$. Thus, we are in the case of a partially enlighted integration domain as in Case 1. Setting again $N=32$, we obtain the following mean ranks for $\mathbf{A}_{T T}$ and $\mathbf{A}_{Q T T}$. As we can see above small distances between the triangles $\tau$ and $\tilde{\tau}$ do not have an

\begin{tabular}{|c|c|c|c|c|}
\hline Accuracy & Mean rank of $\mathbf{A}_{T T}$ & $E_{G, T T}$ & Mean rank of $\mathbf{A}_{Q T T}$ & $E_{G, Q T T}$ \\
\hline $10^{-2}$ & 5.5 & $4 \cdot 10^{-3}$ & 7.4 & $1 \cdot 10^{-3}$ \\
$10^{-3}$ & 9.1 & $2 \cdot 10^{-4}$ & 13.6 & $6 \cdot 10^{-4}$ \\
$10^{-4}$ & 13.8 & $2 \cdot 10^{-6}$ & 22.1 & $4 \cdot 10^{-5}$ \\
$10^{-5}$ & 20.0 & $9 \cdot 10^{-7}$ & 33.2 & $5 \cdot 10^{-7}$ \\
$10^{-6}$ & 27.4 & $1 \cdot 10^{-8}$ & 46.0 & $3 \cdot 10^{-8}$ \\
\hline
\end{tabular}

Table 4.7.

influence on the compression rates of the TT- and QTT approximation and that the ranks are comparable to those in Case 1. Note however that the number of Gauss points $N$ usually has to be chosen larger for such near field integrals in order to preserve a certain accuracy of the quadrature rule (cf. [25]).

As in Case 1, $E_{G, T T}$ and $E_{G, Q T T}$ are quickly decreasing such that a relatively low accuracy of $\mathbf{A}_{T T}$ and $\mathbf{A}_{Q T T}$ is sufficient for the quadrature. The computing times for $Q_{T T}$ and $Q_{Q T T}$ are very similar to those in Case 1 and we therefore refrain from listing them here.

\subsection{Case 5: Higher order basis functions in time}

At last we examine the case of a higher order of the basis functions than considered before. Therefore we adopt the setting in Case 1, i.e., we use the time grid (4.4) and set $c_{\text {shift }}=4.4$. 


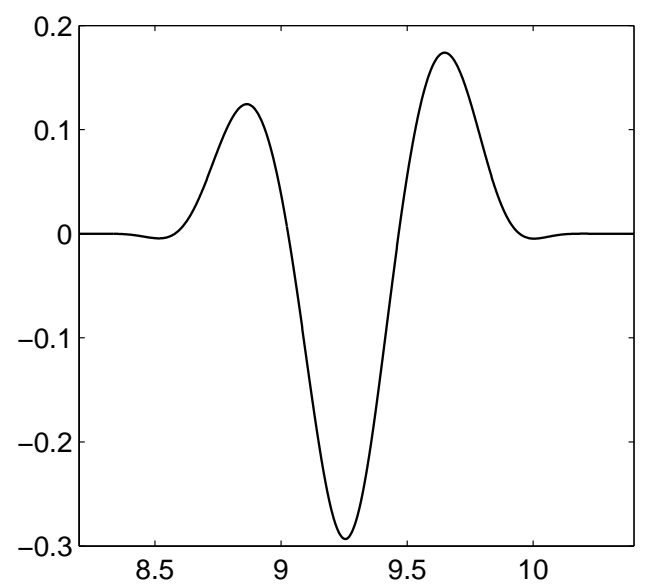

Figure 4.8. Plot of $\psi_{\text {high,1 }}$

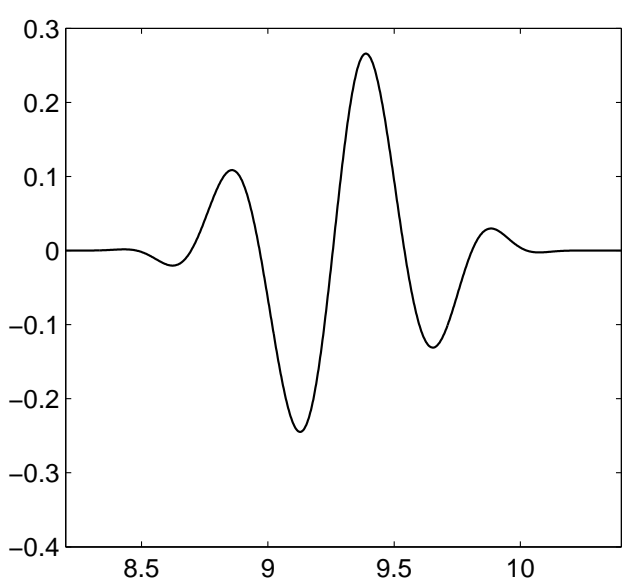

Figure 4.9. Plot of $\psi_{\text {high }, 2}$

Instead of using the basis function in (4.1) we first set

$$
b(t)=\rho_{t_{1}, t_{2}, t_{3}}(t) P_{2}\left(2 \frac{t-t_{1}}{t_{3}-t_{1}}-1\right) \quad \text { and } \quad \tilde{b}(t)=\rho_{t_{4}, t_{5}, t_{6}}(t) P_{3}\left(2 \frac{t-t_{4}}{t_{6}-t_{4}}-1\right),
$$

where $P_{p}$ denotes Legendre polynomials of degree $p$. We denote the corresponding function $\psi$ in (4.3) by $\psi_{\text {high }, 1}$ (cf. Fig. 4.8). As a second example we choose

$$
b(t)=\rho_{t_{1}, t_{2}, t_{3}}(t) P_{5}\left(2 \frac{t-t_{1}}{t_{3}-t_{1}}-1\right) \quad \text { and } \quad \tilde{b}(t)=\rho_{t_{4}, t_{5}, t_{6}}(t) P_{5}\left(2 \frac{t-t_{4}}{t_{6}-t_{4}}-1\right) .
$$

As above we denote the corresponding $\psi$ in (4.3) by $\psi_{\text {high,2 }}$ (cf. Fig. 4.9). In the following we list the mean ranks and the relative errors for both settings.

\begin{tabular}{|c|c|c|c|c|}
\hline Accuracy & Mean rank of $\mathbf{A}_{T T}$ & $E_{G, T T}$ & Mean rank of $\mathbf{A}_{Q T T}$ & $E_{G, Q T T}$ \\
\hline $10^{-2}$ & 4.7 & $4 \cdot 10^{-3}$ & 7.0 & $3 \cdot 10^{-3}$ \\
$10^{-3}$ & 8.5 & $6 \cdot 10^{-5}$ & 13.3 & $2 \cdot 10^{-4}$ \\
$10^{-4}$ & 12.5 & $4 \cdot 10^{-5}$ & 22.1 & $6 \cdot 10^{-5}$ \\
$10^{-5}$ & 18.3 & $6 \cdot 10^{-6}$ & 32.2 & $1 \cdot 10^{-5}$ \\
$10^{-6}$ & 24.7 & $6 \cdot 10^{-7}$ & 44.6 & $1 \cdot 10^{-6}$ \\
\hline
\end{tabular}

Table 4.8.

The table above shows the results for case $\psi_{\text {high, } 1}$. As we can see the mean ranks are not affected by the higher order of the basis functions in this example. They are even slightly lower than in Case 1 . This is due to the fact that $\psi_{\text {high, } 1}$ is not considerably more oscillating than $\psi$ in Case 1 even though Legendre polynomials of higher order are involved. In order to see a negative effect of higher order basis function we have to consider Legendre polynomials of degree 5 , i.e. $\psi_{\text {high,2 }}$, as the next table shows. Also here we can see that the compression rates are not considerably worse than before or in Case 1 even though $\psi_{\text {high,2 }}$ is more oscillatory now. A negative aspect that becomes evident, however, is the slower decrease of $E_{G, T T}$ and $E_{G, Q T T}$. 


\begin{tabular}{|c|c|c|c|c|}
\hline Accuracy & Mean rank of $\mathbf{A}_{T T}$ & $E_{G, T T}$ & Mean rank of $\mathbf{A}_{Q T T}$ & $E_{G, Q T T}$ \\
\hline $10^{-2}$ & 5.5 & $5 \cdot 10^{-1}$ & 9.1 & $6 \cdot 10^{-1}$ \\
$10^{-3}$ & 10.7 & $1 \cdot 10^{-2}$ & 16.8 & $3 \cdot 10^{-3}$ \\
$10^{-4}$ & 14.3 & $1 \cdot 10^{-3}$ & 26.8 & $7 \cdot 10^{-5}$ \\
$10^{-5}$ & 20.8 & $4 \cdot 10^{-5}$ & 37.7 & $2 \cdot 10^{-5}$ \\
$10^{-6}$ & 27.5 & $1 \cdot 10^{-5}$ & 50.9 & $2 \cdot 10^{-5}$ \\
$10^{-7}$ & 44.3 & $5 \cdot 10^{-7}$ & 77.6 & $1 \cdot 10^{-6}$ \\
\hline
\end{tabular}

Table 4.9 .

\subsection{Example on QTT-cross approximation}

As it was mentioned in Remark 3.2 the rank- $r$ QTT-cross approximation takes the advantage of the log-volume cost $O\left(r^{4} \log N\right)$ requiring an evaluation of only $O\left(r^{2} \log N\right) \ll N^{4}$ entries. In the following we give the numerical illustration on QTT-cross approximation for Case 1 above. The next table presents the results of $\varepsilon$-QTT-cross approximation of the target tensor $\mathbf{A}$ of size $32 \times 32 \times 32 \times 32$. We give the CPU time (sec.), QTT and TT $\varepsilon$-ranks and the relative storage size for the obtained TT and QTT approximations. In all cases the storage cost of QTT representation is lower than those for the TT-format. Finally we

\begin{tabular}{|c|c|c|c|}
\hline$\varepsilon$ & $10^{-6}$ & $10^{-5}$ & $10^{-4}$ \\
\hline Time (sec.) & 10.4 & 6.3 & 3.1 \\
QTT-rank & 31 & 21 & 14 \\
TT-rank & 18 & 13 & 9 \\
stor(TT)/stor(QTT) & 1.14 & 1.17 & 1.24 \\
\hline
\end{tabular}

Table 4.10.

notice that the numerical evaluation of the full tensor $\mathbf{A}$ amounts to 321 seconds. For the evaluation of the tensor entries we approximated $\psi$ in (4.3) using Gauss quadrature with 100 points. This high order of approximation is necessary in order to maintain the smoothness of the integrand which is crucial for the good compression rates observed above. Note that an accurate approximation of the 1-dimensional function $\psi(s)$ on a suitable interval by simpler functions, e.g., (piecewise) polynomials, could lead to a further reduction of the computing times.

\section{Conclusion}

In this paper, we have presented a new method for the efficient evaluation of the integrals which arise from the direct discretization of retarded potential integral operators. Since the integrands are $C^{\infty}$ but, in general, not analytic the number of quadrature points is relatively large while the total number of such integrals is huge during the generation of the system matrix. We have introduced the TT and the QTT representations for the fourdimensional quadrature tensors arising from the evaluation of the (transformed) integrands at the quadrature points in the four-dimensional unit cube. We have systematically tested the sensitivity of the algorithm with respect a) to different cases how the smeared discrete 
light cone intersects the spatial mesh, b) to the distance of the surface panels inducing different nearly-singular behaviors of the integrands, and c) to the polynomial degree of the temporal approximation. In all cases the compression by the TT and QTT representation is impressive.

Since both, the TT and the QTT formats require as input the full tensor it is important to substitute the corresponding full-to-TT and full-to-QTT approximation algorithms by their adaptive cross versions. We have performed numerical experiments which show that the compression rates by the adaptive TT-cross and QTT-cross representations are comparable with the original ones while the generation of the full tensor can be avoided.

Acknowledgements. The authors are thankful to Dr. I. Oseledets (INM RAS, Moscow) for the assistance with the QTT-cross-approximation MATLAB routine.

\section{References}

[1] J. Ballani, Fast evaluation of singular BEM integrals based on tensor approximations, Preprint 77/2010, MPI MiS, Leipzig 2010.

[2] A. Bamberger and T. Ha Duong, Formulation Variationnelle Espace-Temps pur le Calcul par Potientiel Retardé de la Diffraction d'une Onde Acoustique, Math. Meth. in the Appl. Sci., 8 (1986), pp. 405-435.

[3] L. Banjai, Multistep and multistage convolution quadrature for the wave equation: Algorithms and experiments, Preprint 58/2009, MPI Leipzig, accepted for publication in SISC.

[4] L. Banjai and S. Sauter, Rapid solution of the wave equation in unbounded domains, SIAM Journal on Numerical Analysis, 47 (2008), pp. 227-249.

[5] L. Banjai and M Schanz, Wave Propagation Problems treated with Convolution Quadrature and BEM, Preprint 60/2010, MPI Leipzig.

[6] Y. Ding, A. Forestier, and T. Ha Duong, A Galerkin scheme for the time domain integral equation of acoustic scattering from a hard surface, The Journal of the Acoustical Society of America, 86 (1989), no. 4, pp. 1566-1572.

[7] J. El Gharib, Problèmes de potentiels rétardes pour l'acoustique, PhD thesis, École Polytechnique, 1999.

[8] T. Ha-Duong, On retarded potential boundary integral equations and their discretisation, In Topics in Computational Wave Propagation: Direct and Iverse Problems, volume 31 of Lect. Notes Comput. Sci. Eng., pp. 301-336, Springer, Berlin, 2003.

[9] T. Ha-Duong, B. Ludwig, and I. Terrasse, A Galerkin BEM for transient acoustic scattering by an absorbing obstacle, International Journal for Numerical Methods in Engineering, 57 (2003), pp. 18451882.

[10] W. Hackbusch, B.N. Khoromskij, S. Sauter, and E. Tyrtyshnikov, Use of Tensor Formats in Elliptic Eigenvalue Problems, Preprint 78, MPI MiS, Leipzig 2008, Numer. Lin. Alg. Appl., 2011.

[11] W. Hackbusch, W. Kress, and S. Sauter, Sparse convolution quadrature for time domain boundary integral formulations of the wave equation by cutoff and panel-clustering, In M. Schanz and O. Steinbach, editors, Boundary Element Analysis, pp. 113-134, Springer, 2007.

[12] W. Hackbusch, W. Kress, and S. Sauter, Sparse convolution quadrature for time domain boundary integral formulations of the wave equation, IMA, J. Numer. Anal., 29 (2009), pp. 158-179.

[13] W. Hackbusch and S. Kühn, A new scheme for the tensor representation, J. of Fourier analysis and applications, 15 (2009), pp. 706-722.

[14] S. Holtz, T. Rohwedder, and R. Schneider, On manifold of tensors of fixed TT-rank, Technical Report 61, TU Berlin, 2010.

[15] B.N. Khoromskij, Introduction to Tensor Numerical Methods in Scientific Computing, Lecture Notes, University/ETH Zürich, Preprint 06-2011, Universität Zürich 2011, pp. 1-238. 
[16] B.N. Khoromskij, Tensors-structured Numerical Methods in Scientific Computing: Survey on Recent Advances, Chemometrics and Intelligent Lab. System, Preprint 21/2010, MPI MIS Leipzig 2010.

[17] B.N. Khoromskij, $O(d \log N)$-Quantics Approximation of $N$-d Tensors in High-Dimensional Numerical Modeling, Constructive Approximation, 34 (2011), no. 2, pp. 257-289, Preprint 55/2009 MPI MIS, Leipzig 2009.

[18] B.N. Khoromskij, V. Khoromskaia, and H.-J. Flad, Numerical Solution of the Hartree-Fock Equation in Multilevel Tensor-structured Format, SIAM J. on Sci. Comp., 33 (2001), no. 1, pp. 45-65.

[19] B.N. Khoromskij and I. Oseledets, Quantics-TT approximation of elliptic solution operators in higher dimensions, Preprint MPI MIS 79/2009, Leipzig 2009. To appear in Rus. J. of Numerical Math., 2010.

[20] T.G. Kolda and B.W. Bader, Tensor decompositions and applications, SIAM Review, 51 (2009), no. 3, pp. $455-500$.

[21] J.C. Nédélec, T. Abboud, and J. Volakis, Stable solution of the retarded potential equations, Applied Computational Electromagnetics Society (ACES) Symposium Digest, 17th Annual Review of Progress, Monterey, 2001.

[22] I.V. Oseledets, Approximation of $2^{d} \times 2^{d}$ matrices using tensor decomposition, SIAM J. Matrix Anal. Appl., 31 (2010), no. 4, pp. 2130-2145.

[23] I.V. Oseledets and E.E. Tyrtyshnikov, Breaking the Curse of Dimensionality, or How to Use SVD in Many Dimensions, SIAM J. Sci. Comp., 31, (2009), pp. 3744-3759.

[24] I.V. Oseledets and E.E. Tyrtyshnikov, TT-cross approximation for multidimensional arrays, Lin. Alg. and its Applications, 432 (2010), pp. 70-88.

[25] S. Sauter and C. Schwab, Boundary Element Methods, Springer, Heidelberg, 2010.

[26] S. Sauter and A. Veit, Adaptive Time Discretization for Retarded Potentials, Preprint 04-2011, Universität Zürich.

[27] S. Sauter and A. Veit, Explicit Solutions of Retarded Boundary Integral Equations, Preprint 03-2011, Universität Zürich.

[28] S. A. Sauter, Über die effiziente Verwendung des Galerkinverfahrens zur Lösung Fredholmscher Integralgleichungen, PhD thesis, Inst. f. Prakt. Math., Universität Kiel, 1992.

[29] EP. Stephan, M. Maischak, and E. Ostermann, Transient boundary element method and numerical evaluation of retarded potentials, In Computational Science - ICCS 2008, pp. 321-330, Springer (5102), 2008.

[30] G. Vidal, Efficient classical simulation of slightly entangled quantum computations, Phys. Rev. Lett., 91 (2003), no. 14, 147902-1 147902-4.

[31] H. Wang and M. Thoss, Multilayer formulation of the multiconfiguration time-dependent Hartree theory, J. Chem. Phys., 119 (2003), pp. 1289-1299.

[32] D. S. Weile, G. Pisharody, N. W. Chen, B. Shanker, and E. Michielssen, A novel scheme for the solution of the time-domain integral equations of electromagnetics, IEEE Transactions on Antennas and Propagation, 52 (2004), pp. 283-295.

[33] D.S. Weile, A.A. Ergin, B. Shanker, and E. Michielssen, An accurate discretization scheme for the numerical solution of time domain integral equations, IEEE Antennas and Propagation Society International Symposium, 2 (2000), pp. 741-744.

[34] D.S. Weile, B. Shanker, and E. Michielssen, An accurate scheme for the numerical solution of the time domain electric field integral equation, IEEE Antennas and Propagation Society International Symposium, 4 (2001), pp. 516-519.

[35] S.R. White, Density-matrix algorithms for quantum renormalization groups, Phys. Rev. B, 48 (1993), no. 14 , pp. $10345-10356$. 
[36] A. Wildman, G. Pisharody, D. S. Weile, S. Balasubramaniam, and E. Michielssen, An accurate scheme for the solution of the time-domain integral equations of electromagnetics using higher order vector bases and bandlimited extrapolation, IEEE Transactions on Antennas and Propagation, 52 (2004), pp. 29732984 . 